\title{
The Price of Distributed Design in Optimal Control
}

\author{
Jean-Charles Delvenne
}

of the entire system's model, even though its decisions affect only a particular subsystem.

It is easy to identify situations where one would prefer sub-controllers to be designed using merely a subset of the plant model data. Think, for example, of the possible future air-traffic management system known as "Free Flight" [5], where each airline will be able to choose its aircrafts' routes and schedules independently. While these choices will have to be cooperative in order to guarantee good global transportation service, airlines will likely want some information to remain private and each decision algorithm will thus have to be designed using partial models only.

Some control synthesis methods of this type exist $[1,2]$, where each sub-controller resulting in overall stability and/or performance can be designed with very little information about other subsystems. Each mapping $F_{j}$ in Equation (1) depends on the corresponding subsystem's state-space data and additional parameters which capture the influence of its neighbors. (In the case of [2], a complete model of the other subsystems is not required but they must be known to satisfy some type of Integral Quadratic Constraint. For [1], the additional information is the number of neighbors).

From the viewpoint of robustness to failures, privacy protection, and even communication and computation, it would be interesting to be able to design control laws which use as little information as possible about the plant model and/or such that the map $F_{j}$ can be determined using solely a model of the subsystem receiving the input $u_{j}$. Indeed, even if they are not distributed in the classical sense explained above, such control laws are modular, because each subsystem and corresponding sub-controller form a fixed unit, which can be designed by a separate controller-builder, irrespective of the other units it is connected to. It can thus be "plugged-in", turned on and off, or disconnected at will without altering the overall system. The price to pay for this modularity is, of course, a lower level of guaranteed closed-loop performance.

Apart from pointing out this distinction between distributed control laws and distributed control design strategies (which result in modular control laws), the goal of this paper is to study the degradation in performance 
caused by modularity. We show that, for discrete-time linear time-invariant systems with input matrix "B" equal to the identity, the deadbeat strategy, which constructs a controller that stabilizes the given plant in a single time step, enjoys several optimality properties among distributed design strategies.

We consider a distributed design strategy as an algorithm mapping the plant model's data into a controller, and characterize the input/output properties of this map. To this end, we use both the notion of (un)domination from Economics- which compares strategies to each other over all possible inputs- and that of competitive ratio from Computer Science, which compares a given distributed strategy to the optimal one, in the worst case [4].

The following notation will be used throughout the paper. If $\mathbf{A}$ is a subset of $\mathbf{S}$ then $\mathbf{A}^{c}$ is the complement of $\mathbf{A}$ in $\mathbf{S}$, i.e., the set of all elements of $\mathbf{S}$ which do not belong to $\mathbf{A}$. For a matrix $M, M_{j}$ will denote the $j^{\text {th }}$ row of $M$. It is a row-vector. When $M$ is a symmetric matrix, $M \succ 0$ (respectively $M \succeq 0$ ) means that it is positive definite (respectively, positive semi-definite). Finally, $e_{i}$ will denote the column-vector with all entries zero except the $i^{t h}$ one, which is equal to one.

\section{Model and problem statement}

We consider the Linear Quadratic Regulator problem, where the cost function

$$
J\left(\mathrm{x},\{u(i)\}_{i=0}^{\infty}\right)=\sum_{i=1}^{\infty} x(i)^{T} x(i)+\sum_{i=0}^{\infty} u(i)^{T} u(i)
$$

is to be minimized with respect to the choice of control inputs $\{u(i)\}_{i=0}^{\infty}$, along the trajectory of the discrete-time linear time-invariant dynamical system

$$
\begin{aligned}
x(i+1) & =A x(i)+u(i) \text { for all } i \geq 0, \\
x(0) & =\mathrm{x} \in \mathbb{R}^{n} .
\end{aligned}
$$

Note that, when defining the cost function $J$ in (2), we started the summation on $x$ at index $i=1$, instead of the more common $i=0$. This was done to obtain cleaner formulae in later developments and is otherwise inconsequential (since the term $x(0)^{T} x(0)$ only contributes a constant, independent of the choice of inputs).

As explained in the introduction, we are interested in static state feedback solutions which can be constructed by separate controller-builders. More precisely, as a first approach, we are looking for controls $\{u(i)\}_{i=0}^{\infty}$ of the form

$$
u(i)=K x(i),
$$

where each row $K_{j}$ of the controller's $n \times n$ gain matrix $K$ depends only on the corresponding row $A_{j}$ of the $n \times n$ plant matrix $A$. When this condition is satisfied, we will say that the control law is modular and that it is designed using a distributed design strategy.

For a given design strategy $\mathscr{S}$, plant matrix $A$ and initial condition $\mathrm{x}$, we will denote the resulting controller by $K_{\mathscr{S}}(A)$, the corresponding control law by $u_{\mathscr{S}(A)}$, and the achieved performance by $\mathscr{S}(A, \mathrm{x})$, i.e.,

$$
\mathscr{S}(A, \mathrm{x}):=J\left(\mathrm{x},\left\{u_{\mathscr{S}(A)}(i)\right\}_{i=0}^{\infty}\right) .
$$

The optimal design strategy, denoted by opt, is the one which, to every plant matrix $A$ associates the control law that minimizes the cost $J$. Its controller matrix $K_{\text {opt }}(A)$ and performance $\operatorname{opt}(A, \mathrm{x})$ can be computed after solving a Riccati equation involving $A$.

Our goal, in the remainder of this paper, is to give bounds on the closed-loop performance that can be achieved by a modular strategy. Before doing so, we end this section with an easy result which we will use repeatedly.

Proposition 1 Let $\mathscr{S}$ be a design strategy (distributed or not). Then, for all $A$ and $x$,

$$
\begin{aligned}
& \mathscr{S}(A, x)=x^{T}\left[K_{\mathscr{S}}(A)^{T} K_{\mathscr{S}}(A)+\right. \\
& \left.\sum_{i=1}^{\infty}\left(\left(A+K_{\mathscr{S}}(A)\right)^{i}\right)^{T}\left(I+K_{\mathscr{S}}(A)^{T} K_{\mathscr{S}}(A)\right)\left(A+K_{\mathscr{S}}(A)\right)^{i}\right] x,
\end{aligned}
$$

where the series converge if and only if $\left(A+K_{\mathscr{S}}(A)\right)$ is stable. In addition, every term in the series is a positive semi-definite matrix and, thus, any partial sum gives a lower bound on $\mathscr{S}(A, x)$.

\section{The price of modularity}

In this section, we show that the requirement that a design strategy be distributed strongly affects the performance of the obtained controller.

\subsection{Competitive ratio and the deadbeat strategy}

The notion of competitive ratio first appeared in the Computer Science literature as a way to gauge the performance of on-line algorithms [3]. It was later applied to distributed optimization algorithms [4]. Building on these ideas, we define the competitive ratio of a distributed control design strategy $\mathscr{S}$ as

$$
R(\mathscr{S}):=\sup _{A, x} \frac{\mathscr{S}(A, x)}{\operatorname{opt}(A, x)},
$$

where we make the convention that " $\frac{0}{0}$ " equals one.

In words, we are comparing the performance (as measured by the cost $J$ ) of a distributed strategy over all possible instances of the controller design problem (defined by the matrix $A$ and initial conditions $x$ ) to that 
of the optimal control strategy. The competitive ratio of any design strategy (even a non-distributed one) is always greater than one and a small ratio indicates a strategy with performance uniformly close to that of the optimal controller. If we can determine distributed strategies with minimal competitive ratio, i.e., if we can solve the following problem

$$
\underset{\mathscr{d i s t r i b u t e d}}{\operatorname{argmin}} R(\mathscr{S})
$$

we will thus have a measure of the intrinsic performance degradation due to the segmentation of information between several controller-builders.

We start by introducing the so-called deadbeat strategy $\mathscr{D}$, defined by

$$
K_{\mathscr{D}}(A):=-A \text { for all matrix } A .
$$

As the name indicates, the deadbeat control design strategy generates a controller which brings the state of any system of the form (3) to the origin in a single time-step. Using this property, it is easy to see that the cost of the deadbeat strategy for all matrix $A$ and initial condition $x$ is simply

$$
\mathscr{D}(A, x)=x^{T} A^{T} A x .
$$

Note that, for every plant matrix $A$, the control law $u_{\mathscr{D}(A)}$ is both modular and distributed in the classical sense, since $K_{\mathscr{D}}(A)$ has the same sparsity structure as $A$. The properties of strategy $\mathscr{D}$ allow to compute its competitive ratio exactly.

Proposition 2 The deadbeat design strategy $\mathscr{D}$ has competitive ratio 2 .

Proof: Let us start with bounding the performance of strategy $\mathscr{D}$. For this purpose, consider the optimal (centralized) design strategy opt whose performance is given by $\operatorname{opt}(A, x)=x^{T}(P-I) x$ where, (see, e.g., [6]), for any $A$, matrix $P \succ 0$ is the only positive-definite solution of the Riccati equation

$$
P=A^{T} P A-A^{T} P(I+P)^{-1} P A+I,
$$

and the corresponding gain matrix is

$$
K_{o p t}(A)=-(I+P)^{-1} P A .
$$

By definition of the cost $J$, we see that

$P-I \succeq K_{o p t}(A)^{T} K_{o p t}(A)+\left(A+K_{o p t}(A)\right)^{T}\left(A+K_{o p t}(A)\right)$.

Expanding the right-hand side and using (5), we obtain

$P-I \succeq$

$A^{T}\left[P(I+P)^{-2} P+\left(I-(I+P)^{-1} P\right)^{T}\left(I-(I+P)^{-1} P\right)\right] A$
Let us denote the right-hand side of (6) by $A^{T} g(P) A$. Then we can make the following two claims regarding the rational function $g$.

Claim 1 For any scalar $p>0, g(p)=1-\frac{2 p}{(1+p)^{2}}$ and $g(p) \geq \frac{1}{2}$.

Claim 2 Let $P \succ 0$ be a positive-definite matrix and $\Lambda, T$ be diagonal and orthogonal matrices, respectively, such that $P=T^{T} \Lambda T$. Then $g(P)=T^{T} \operatorname{diag}\left(g\left(\lambda_{i}\right)\right) T$, where the $\lambda_{i}$ 's are the diagonal elements of $\Lambda$ (and the eigenvalues of $P$ ).

Claim 1 is proved by computing the minimum of $g$ over $[0, \infty)$, while Claim 2 follows from the fact that all matrices involved in the computation of $g$ can be diagonalized in the same basis. Using these two claims, we find that, for all $A$

$$
\begin{aligned}
P-I & \succeq A^{T} g(P) A \\
& =A^{T} T^{T} \operatorname{diag}\left(g\left(\lambda_{i}\right)\right) T A \quad(\text { by Claim } 2) \\
& \succeq \frac{1}{2} A^{T} T^{T} T A \quad(\text { by Claim } 1) \\
& =\frac{1}{2} A^{T} A .
\end{aligned}
$$

Hence, for all $A$ and $x, \operatorname{opt}(A, x) \geq \frac{1}{2} \mathscr{D}(A, x)$ and, taking the sup over $A$ and $x$, we obtain

$$
R(\mathscr{D}) \leq 2
$$

The fact that there is equality in inequality (7) is a direct consequence of Lemma 1, which will prove useful again later.

\section{Lemma 1 Consider the nilpotent $n \times n$ matrix}

$$
A=\left[\begin{array}{cccc}
0 & \ldots & 0 & r \\
\vdots & \ddots & & 0 \\
\vdots & & \ddots & \vdots \\
0 & \ldots & \ldots & 0
\end{array}\right]
$$

Then, for all $x$, opt $(A, x)=\frac{1}{2} x^{T} A^{T} A x=\frac{1}{2} \mathscr{D}(A, x)$.

Proof: The proof consists in showing that $I+\frac{1}{2} A^{T} A$ satisfies the Riccati equation. This follows from simple algebra, using the fact that $A^{2}=0$ and $\left(2 I+\frac{1}{2} A^{T} A\right)^{-1} e_{1}=$ $\frac{1}{2} e_{1}$.

The second result characterizes the best performance that can be achieved by the output of any distributed strategy.

Proposition 3 No distributed design strategy has a competitive ratio strictly smaller than 2 . 
Proof: First note that if design strategy $\mathscr{S}$ is such that $\mathscr{S}(0, x) \neq 0$ for some $x$, then it has unbounded competitive ratio. Because

$$
\mathscr{S}(0, x) \geq x^{T} K_{\mathscr{S}}(0)^{T} K_{\mathscr{S}}(0) x \text { for all } x,
$$

this means that strategy $\mathscr{S}$ has bounded competitive ratio only if $K_{\mathscr{S}}(0)=0$. Consider such a strategy along with the one-parameter families of matrices

$$
\begin{gathered}
U(r)=\left[\begin{array}{cccc}
0 & \ldots & 0 & r \\
\vdots & \ddots & & 0 \\
\vdots & & \ddots & \vdots \\
0 & \ldots & \ldots & 0
\end{array}\right], \\
V(r)=\left[\begin{array}{cccc}
0 & \ldots & 0 & r \\
\vdots & \ddots & & 0 \\
\vdots & & \ddots & \vdots \\
r & \ldots & \ldots & 0
\end{array}\right] \\
W(r)=\left[\begin{array}{cccc}
0 & \ldots & 0 & 0 \\
\vdots & \ddots & & 0 \\
\vdots & & \ddots & \vdots \\
r & \ldots & \ldots & 0
\end{array}\right] .
\end{gathered}
$$
have

Since $\mathscr{S}$ is a distributed strategy and $K_{\mathscr{S}}(0)=0$, we

$$
\begin{aligned}
K_{\mathscr{S}}(U(r))= & {\left[\begin{array}{ccc}
a_{1}(r) & \ldots & a_{n}(r) \\
0 & \ldots & 0 \\
\vdots & & \vdots \\
0 & \ldots & 0
\end{array}\right], } \\
K_{\mathscr{S}}(W(r))= & {\left[\begin{array}{ccc}
0 & \ldots & 0 \\
0 & \ldots & 0 \\
\vdots & & \vdots \\
b_{1}(r) & \ldots & b_{n}(r)
\end{array}\right], } \\
K_{\mathscr{S}}(V(r))= & {\left[\begin{array}{ccc}
a_{1}(r) & \ldots & a_{n}(r) \\
0 & \ldots & 0 \\
\vdots & & \vdots \\
b_{1}(r) & \ldots & b_{n}(r)
\end{array}\right] . }
\end{aligned}
$$

Note that, to have a bounded competitive ratio, strategy $\mathscr{S}$ must also be stabilizing, i.e., $A+K_{\mathscr{S}}(A)$ is stable for all matrix $A$ (for, otherwise, it would yield an infinite cost for some matrices while the corresponding optimal cost remains bounded since the pair $(A, I)$ is controllable for any matrix $A$ ). Hence, in particular, the matrices

$$
U^{c l}(r)=\left[\begin{array}{ccc}
a_{1}(r) & \ldots & a_{n}(r)+r \\
0 & \ldots & 0 \\
\vdots & & \vdots \\
0 & \ldots & 0
\end{array}\right]
$$

$$
\begin{aligned}
W^{c l}(r)= & {\left[\begin{array}{ccc}
0 & \ldots & 0 \\
0 & \ldots & 0 \\
\vdots & & \vdots \\
b_{1}(r)+r & \ldots & b_{n}(r)
\end{array}\right], } \\
\text { and } V^{c l}(r)= & {\left[\begin{array}{ccc}
a_{1}(r) & \ldots & a_{n}(r)+r \\
0 & \ldots & 0 \\
\vdots & & \vdots \\
b_{1}(r)+r & \ldots & b_{n}(r)
\end{array}\right] . }
\end{aligned}
$$

are stable for all $r$.

This implies that

$$
\begin{gathered}
\left|a_{1}(r)\right|<1,\left|b_{n}(r)\right|<1 \text {, and } \\
\left|a_{1}(r) b_{n}(r)-\left(r+b_{1}(r)\right)\left(r+a_{n}(r)\right)\right|<1 \text {, for all } r .
\end{gathered}
$$

The last inequality follows from the fact that the spectrum of $V^{c l}(r)$ consists of 0 (with geometric multiplicity $n-2$ ) and of the two eigenvalues of matrix

$$
\left[\begin{array}{cc}
a_{1}(r) & a_{n}(r)+r \\
b_{1}(r)+r & b_{n}(r)
\end{array}\right] .
$$

As a result, we see that $\left|\left(r+b_{1}(r)\right)\left(r+a_{n}(r)\right)\right|<2$ and, thus, that

$$
\left|r+b_{1}(r)\right|<\sqrt{2} \text { or }\left|r+a_{n}(r)\right|<\sqrt{2} .
$$

In the remainder of this proof, we will assume, without loss of generality, that

$$
\left|r+a_{n}(r)\right|<\sqrt{2} \text { for all } r .
$$

Now, note that, for all $r$ and vectors $x$

$$
\begin{aligned}
\mathscr{S}(U(r), x) & \geq x^{T} K_{\mathscr{S}}(U(r))^{T} K_{\mathscr{S}}(U(r)) x \\
& =x^{T}\left[\begin{array}{c}
a_{1}(r) \\
\vdots \\
a_{n}(r)
\end{array}\right]\left[\begin{array}{c}
a_{1}(r) \\
\vdots \\
a_{n}(r)
\end{array}\right]^{T} x,
\end{aligned}
$$

while the optimal cost is given by

$$
\operatorname{opt}(U(r), x)=x^{T}\left[\begin{array}{ccc}
0 & & 0 \\
\vdots & \ddots & \vdots \\
0 & & \frac{r^{2}}{2}
\end{array}\right] x
$$

according to Lemma 1. In particular, using (8), we see that, for all $r>0$ large enough

$$
\begin{aligned}
\mathscr{S}\left(U(r), e_{n}\right) & =a_{n}(r)^{2} \\
& >(r-\sqrt{2})^{2} \\
& \geq\left(2-\frac{8}{r}\right) \frac{r^{2}}{2} \\
& =\left(2-\frac{8}{r}\right) \operatorname{opt}\left(U(r), e_{n}\right) .
\end{aligned}
$$


Hence,

$$
\begin{aligned}
R(\mathscr{S}) & \geq \lim _{r \rightarrow \infty} \frac{\mathscr{S}\left(U(r), e_{n}\right)}{\operatorname{opt}\left(U(r), e_{n}\right)} \\
& \geq 2,
\end{aligned}
$$

which finishes the proof.

Collecting the results of Propositions 2 and 3, we obtain the main theorem of this section.

Theorem 1 The deadbeat strategy has a competitive ratio of 2 and no other distributed design strategy has a better competitive ratio.

In other words, Theorem 1 says that the deadbeat strategy is minimal among distributed strategies for the order defined by the competitive ratio and shows that the intrinsic "price of modularity" is a factor of 2 in performance.

\subsection{Undominated design strategies}

The previous section showed that the deadbeat had the best worst-case performance (as measured by the competitive ratio) among all distributed strategies. However, it is a priori possible that other distributed strategies exist which, while having the same worst-case performance, perform better on some instances and never worse. Such strategies would clearly be desirable because they would provide a better trade-off between average and worst-case performance. In this section, we prove that such distributed control design strategies in fact do not exist. We start by making the following definition.

Definition 1 (i) A design strategy $\mathscr{S}$ is said to dominate another strategy $\mathscr{T}$ whenever

$$
\mathscr{S}(A, x) \leq \mathscr{T}(A, x) \text { for all } A, x
$$

and the inequality is strict for at least one pair $(\bar{A}, \bar{x})$.

(ii) A strategy $\mathscr{T}$ is said to be undominated (by strategies in a set $\mathbf{S}$ ) if there does not exist a strategy $\mathscr{S}$ (in the set $\mathbf{S}$ ) which dominates it.

We can now prove another minimality property of the deadbeat. Note that the following result does not rule out the existence of other undominated distributed strategies with competitive ratio two.

Theorem 2 The deadbeat strategy $\mathscr{D}$ is undominated among distributed strategies.

Proof: We are going to prove that, for any distributed strategy $\mathscr{S}$, there exist a matrix $A$ and initial condition $x$ such that $\mathscr{S}(A, x)>\mathscr{D}(A, x)=x^{T} A^{T} A x$. We will proceed in several steps, which require us to partition the set of distributed strategies $\mathbf{D}$ as follows:

$$
\mathbf{D}=\mathbf{C}^{c} \cup \mathbf{S}_{\mathbf{1}} \cup \mathbf{S}_{\mathbf{0}} \cup\{\mathscr{D}\},
$$

where

$$
\begin{aligned}
\mathbf{C}:= & \left\{\text { distributed strategy } \mathscr{S}, \exists \lambda_{j}: \mathbb{R}^{n} \rightarrow \mathbb{R},\right. \\
& \left.\left(K_{\mathscr{S}}(A)\right)_{j}=\lambda_{j}\left(A_{j}\right) A_{j}, \text { for all } j=1 \ldots N\right\}, \text { i.e. },
\end{aligned}
$$

$\mathbf{C}$ is the set of all distributed design strategies for which each row of the designed controller is co-linear with the corresponding row of the plant matrix,

$\mathbf{S}_{\mathbf{1}}:=\left\{\mathscr{S} \in \mathbf{C}, \exists j, i \neq j\right.$ and $\left.a \in \mathbb{R} e_{i}, 1+\lambda_{j}(a) \neq 0\right\}$,

and

$$
\mathbf{S}_{\mathbf{0}}:=\left\{\mathscr{S} \in \mathbf{C} \backslash \mathbf{S}_{\mathbf{1}}, \exists j, a \in \mathbb{R}^{n}, 1+\lambda_{j}(a) \neq 0\right\} .
$$

The result of Theorem 2 then simply follows from the fact that $\mathscr{D}$ is undominated by strategies in $\mathbf{C}^{c}, \mathbf{S}_{\mathbf{1}}$ and $\mathbf{S}_{\mathbf{0}}$. We prove each of these claims in turn.

Proposition 4 The deadbeat is undominated by strategies in $\mathbf{C}^{c}$.

Proof: Let $\mathscr{S} \in \mathbf{C}^{c}$ and let $j$ be such that $\left(K_{\mathscr{S}}(\bar{A})\right)_{j}$ is not co-linear with $\bar{A}_{j}$ for some matrix $\bar{A}$. Let $a^{T}=\bar{A}_{j}$ and consider the matrix $A$ such that the row $A_{j}=a^{T}$ and $A_{i}=0$ for all $i \neq j$.

If $K_{\mathscr{S}}(0) \neq 0$, then $\mathscr{S}$ cannot dominate $\mathscr{D}$ (since $\mathscr{D}(0, x)=0$ for all $x)$ and, thus, there is no loss of generality in assuming that $K_{\mathscr{S}}(0)=0$ and, in turn, that $\left(K_{\mathscr{S}}(A)\right)_{i}=0$ for all $i \neq j$. Let us also denote $K_{\mathscr{S}}(A)$ by $K$ and $\left(K_{\mathscr{S}}(A)\right)_{j}$ by $k^{T}$. For all $x$,

$$
\begin{gathered}
\mathscr{S}(A, x) \geq x^{T}\left(K^{T} K+(A+K)^{T}(A+K)\right) x, \text { and } \\
\mathscr{S}(A, x)-\mathscr{D}(A, x) \geq x^{T}\left(K^{T}(A+K)+(A+K)^{T} K\right) x .
\end{gathered}
$$

Now, note that $K^{T}(A+K)+(A+K)^{T} K=k(a+k)^{T}+$ $(a+k) k^{T}$ and that this rank-2 matrix admits the two scalars $(a+k)^{T} k \pm\|a+k\|\|k\|$ as eigenvalues. (This can be easily shown by looking for eigenvectors of the form $\alpha(a+k)+\beta k$, and computing the values of $\alpha$ and $\beta$.)

By assumption, vectors $a$ and $a+k$ are not co-linear and, thus, by Cauchy-Schwartz's (strict) inequality, $\mid(a+$ $k)^{T} k \mid<\|a+k\|\|k\|$. This shows that one of these eigenvalues is strictly positive. Hence, there exists an $x$ such that

$$
\begin{aligned}
\mathscr{S}(A, x)-\mathscr{D}(A, x) & \geq x^{T}\left[K^{T}(A+K)+(A+K)^{T} K\right] x \\
& >0
\end{aligned}
$$

and strategy $\mathscr{S}$ does not dominate the deadbeat strategy D.

Proposition 5 The deadbeat is undominated by strategies in $\mathbf{S}_{\mathbf{1}}$. 
Proof: $\quad$ Let $\mathscr{S} \in \mathbf{S}_{\mathbf{1}}$ and, for any given indices $i$ and $j, i \neq j$, consider the matrix $A$ defined by $A_{i}=r e_{j}^{T}$, $A_{j}=s e_{i}^{T}$ and all other rows equal to zero, i.e., $A$ has all entries zero, except the $(i, j)^{t h}$-one, which is $r$ and the $(j, i)^{t h}$-one, which is $s$. Then, $\left(K_{\mathscr{S}}(A)\right)_{i}=\lambda_{i}\left(r e_{j}\right) r e_{j}^{T}$, $\left(K_{\mathscr{S}}(A)\right)_{j}=\lambda_{j}\left(s e_{i}\right) s e_{i}^{T}$, and $\left(K_{\mathscr{S}}(A)\right)_{m}=0$ for $m \neq i, j$. As before, we will denote $K_{\mathscr{S}}(A)$ by $K$.

Now,

$$
\begin{aligned}
& \mathscr{S}(A, x)> \\
& x^{T}\left[(A+K)^{T} K^{T} K(A+K)+\left((A+K)^{2}\right)^{T}(A+K)^{2}\right] x,
\end{aligned}
$$

and easy algebra shows that the matrix on the right handside is diagonal with all entries zero except the $(i, i)^{t h}$ and $(j, j)^{t h}$ - ones which are, respectively,

$$
\left(1+\lambda_{j}\left(s e_{i}\right)\right)^{2}\left(\lambda_{i}\left(r e_{j}\right)^{2}+\left(1+\lambda_{i}\left(r e_{j}\right)\right)^{2}\right) r^{2} s^{2},
$$

and

$$
\left(1+\lambda_{i}\left(r e_{j}\right)\right)^{2}\left(\lambda_{j}\left(s e_{i}\right)^{2}+\left(1+\lambda_{j}\left(s e_{i}\right)\right)^{2}\right) r^{2} s^{2} .
$$

Because $\mathscr{S} \in \mathbf{S}_{\mathbf{1}}$, there exists an index $j$ and a scalar $s$ (which, as before, can be assumed to be non-zero without loss of generality since, otherwise, $\mathscr{S}$ cannot dominate the deadbeat) such that $\left(1+\lambda_{j}\left(s e_{i}\right)\right) \neq 0$ for some $i$. Moreover, $\lim _{r \rightarrow \infty}\left(\lambda_{i}\left(r e_{j}\right)^{2}+\left(1+\lambda_{i}\left(r e_{j}\right)\right)^{2}\right) r^{2}=+\infty$, irrespective of the function $\lambda_{i}($.$) .$

Hence, we can always construct $A$ with the appropriate value of $i$ and a scalar $r$ large enough to make the expression in (10) strictly greater than $s^{2}$, i.e., to ensure that $(A+K)^{T} K^{T} K(A+K)+\left((A+K)^{2}\right)^{T}(A+K)^{2}-A^{T} A$ has a strictly positive eigenvalue. As a result, $\mathscr{S}$ does not dominate $\mathscr{D}$.

Proposition 6 The deadbeat is undominated by strategies in $\mathbf{S}_{\mathbf{0}}$.

Proof: Let $\mathscr{S} \in \mathbf{S}_{\mathbf{0}}$ and index $j$ and vector $a$ be such that $\left(1+\lambda_{j}(a)\right) \neq 0$. Consider the matrix $A$ defined by $A_{j}=a^{T}$ and $A_{n}=s e_{1}^{T}, A_{m}=0$ for all $m \neq j, n$ (where, without loss of generality, we have assumed $j \neq n$ ). The scalar $s$ is to be specified later.

Then $\left(A+K_{\mathscr{S}}(A)\right)_{j}=\left(1+\lambda_{j}(a)\right) a^{T} \quad$ and $\left(A+K_{\mathscr{S}}(A)\right)_{m}=0$ for all $m \neq j$ ( and, in particular $m=n$, since $\mathscr{S}$ does not belong to $\mathbf{S}_{\mathbf{1}}$ ). Again, $K$ will stand for $K_{\mathscr{S}}(A)$. Then, easy algebra shows that

$$
\begin{aligned}
& K^{T} K+(A+K)^{T} K^{T} K(A+K)-A^{T} A= \\
& \left(\lambda_{j}(a)^{2}+\left(1+\lambda_{j}(a)\right)^{2} s^{2}+\lambda_{j}(a)^{2}\left(1+\lambda_{j}(a)\right)^{2} a(1)^{2}-1\right) a a^{T}
\end{aligned}
$$

and hence, since $\left(1+\lambda_{j}(a)\right) \neq 0$, we can choose $s$ large enough to ensure that this matrix has a strictly positive eigenvalue. When $A$ is constructed with such a value of $s$, there exists an initial condition $x$ such that $\mathscr{S}(A, x)>$ $\mathscr{D}(A, x)$ and thus, strategy $\mathscr{S}$ does not dominate $\mathscr{D}$.

\section{Conclusion and future work}

We have studied distributed control design strategies and modular control laws and quantified their intrinsic performance limitations for discrete-time systems that can be brought to the origin in a single time step. As this class of systems is clearly restricted, it would be interesting to extend the study of the limitations of distributed design to more general plant models. The role of deadbeat strategies may be less significant for discrete-time systems with general input matrix, and is meaningless in the case of continuous-time systems.

Even within the restricted framework of this paper, there are several directions in which this work could be naturally extended. For instance, one could study cases where, instead of depending on a given row, each $\left(K_{\mathscr{S}}(A)\right)_{j}$ depends on some other subset of entries of matrix $A$. One could also consider additional constraints on control design strategies and similarly study the ensuing performance limitation. The requirement that each controller-builder does not know which row of the matrix $K_{\mathscr{S}}(A)$ it is designing (or, in the words of [4], that the strategy be homogeneous) would be particularly interesting in this respect, as it would result in even more modularity, at the expense of a possibly larger minimal competitive ratio.

Finally, one could also ask the following question: "What is the minimum amount of information about $A$ that each controller-builder needs to know to construct the row $\left(K_{\mathscr{S}}(A)\right)_{j}$ of a controller with closed-loop performance $(1+\varepsilon) \operatorname{opt}(A, x)$, for $0 \leq \varepsilon<1$ ?"

\section{References}

[1] R. Cogill and S. Lall. Control design for topologyindependent stability of interconnected systems. In Proc. of the American Control Conference, pp. 3717-3722, 2004.

[2] C. Langbort, R. S. Chandra and R. D'Andrea. Distributed Control Design for Systems Interconnected over an Arbitrary Graph. IEEE Transactions on Automatic Control, vol. 49, no. 9, pp. 1502-1519, Sept 2004.

[3] M.S. Manasse, L.A. McGeoch, and D.D. Sleator. Competitive algorithms for on-line problems. In Proc. of the $20^{\text {th }}$ ACM Symposium on Theory of Computing, pp. 322-333, 1988.

[4] C.H. Papadimitriou, and M. Yannakakis. Linear programming without the matrix. In Proc. of the $25^{\text {th }}$ ACM Symposium on Theory of Computing, pp. 121-127, 1993.

[5] T.S. Perry. In search of the future of air-traffic control. IEEE Spectrum, vol. 34, no. 8, pp. 18-35, 1997.

[6] R.F. Stengel. Optimal Control and Estimation, Dover Publications, 1994. 